\title{
A Rare Case of Mycobacterium abscessus Cardiac Resynchronization Therapy Defibrillator (CRT-D) Device Infection
}

\author{
Adam S Potter, MD, PhD ${ }^{1^{*}}$, Brittany Duong, DO', Abraham Lee, DO', Bao X Nguyen, DO ${ }^{1}$, David \\ Reynoso, MD, PhD ${ }^{3}$ and Asif A Sewani, $M^{1}$
}

${ }^{1}$ Division of Cardiology, Heart Rhythm Center, The University of Texas Medical Branch, USA

${ }^{2}$ Department of Internal Medicine, The University of Texas Medical Branch, USA

${ }^{3}$ Division of Infectious Diseases, The University of Texas Medical Branch, USA

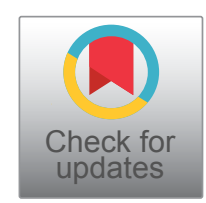

*Corresponding author: Adam S Potter, MD, PhD, Division of Cardiology, Heart Rhythm Center, The University of Texas Medical Branch, Galveston, Texas 77555, USA, Tel: 409-772-1533, Fax: 409-772-4982

\section{Keywords}

Cardiac resynchronization therapy, Endocarditis, Electrophysiology

\section{Abbreviations \\ CABG: Coronary Artery Bypass Graft; TEE: Transesopha- geal Echocardiogram; PCl: Percutaneous Intervention; HFrEF: Heart Failure with Reduced Ejection Fraction; DVT: Deep Vein Thrombosis}

\section{Introduction}

Rapid-growing Mycobacteria comprise approximately half of the currently known mycobacterial species and are divided into five major groups: Mycobacterium fortuitum, M. chelonae/M. abscessus complex, $M$. smegmatis, $M$. mucogenicum, and the pigmented rapidly-growing Mycobacteria. In clinical practice, the most commonly encountered species are $M$. fortuitum and $M$. chelonae/abscessus [1,2]. These infections are geographically related to non-sterile water and soil reservoirs, especially in the southern United States [3]. The most common mode of transmission is inhalation and direct contact with water and soil. Disseminated disease with rapid-growers can be seen in patients with predisposing deficiencies of cell-mediated immunity. Infections of implantable cardiac devices by rapidly-growing, non-tuberculosis mycobacteria are relatively rare, with only 28 documented cases in the English literature, and only two of the 28 cases involving Myco- bacterium abscessus, highlighting the singularity of this case [4].

\section{Case Report}

A 57-year-old male presented to the ED with fever, night sweats, erythema and slight purulent drainage from his CRT-D pocket incision 12 days after having the device placed. The patient was transferred to our facility with concern for device pocket infection and electrophysiology evaluation. On initial presentation, patient appeared to be in moderate discomfort; he was febrile to 102.7 degrees Fahrenheit, blood pressure was $118 / 65$, and pulse was 95 bpm. Physical exam revealed a tender and warm device pocket incision with purulent drainage, and surrounding erythema, induration, and ecchymosis extending into the axilla, without lymphadenopathy (Figure 1).

The patient's medical history was significant for coronary artery disease with CABG in 2009 and multiple PCls, chronic HFrEF despite $>1$ year on goal directed medical therapy, hypertension, and hyperlipidemia.

The differential diagnosis for the patient's fever, night sweats, and chills includes infection from multiple possible etiologies, including surgical site infection, implantable cardiac device pocket infection, infective endocarditis involving the newly placed CRT-D leads, post-procedural nosocomial infections such as Clostridioides difficile colitis, deep venous thrombosis, mali-

Citation: Potter AS, Duong B, Lee A, Nguyen BX, Reynoso D, et al. (2021) A Rare Case of Mycobacterium abscessus Cardiac Resynchronization Therapy Defibrillator (CRT-D) Device Infection. Int J Clin Cardiol 8:231. doi.org/10.23937/2378-2951/1410231

Accepted: July 01, 2021: Published: July 03, 2021

Copyright: (c) 2021 Potter AS, et al. This is an open-access article distributed under the terms of the Creative Commons Attribution License, which permits unrestricted use, distribution, and reproduction in any medium, provided the original author and source are credited. 


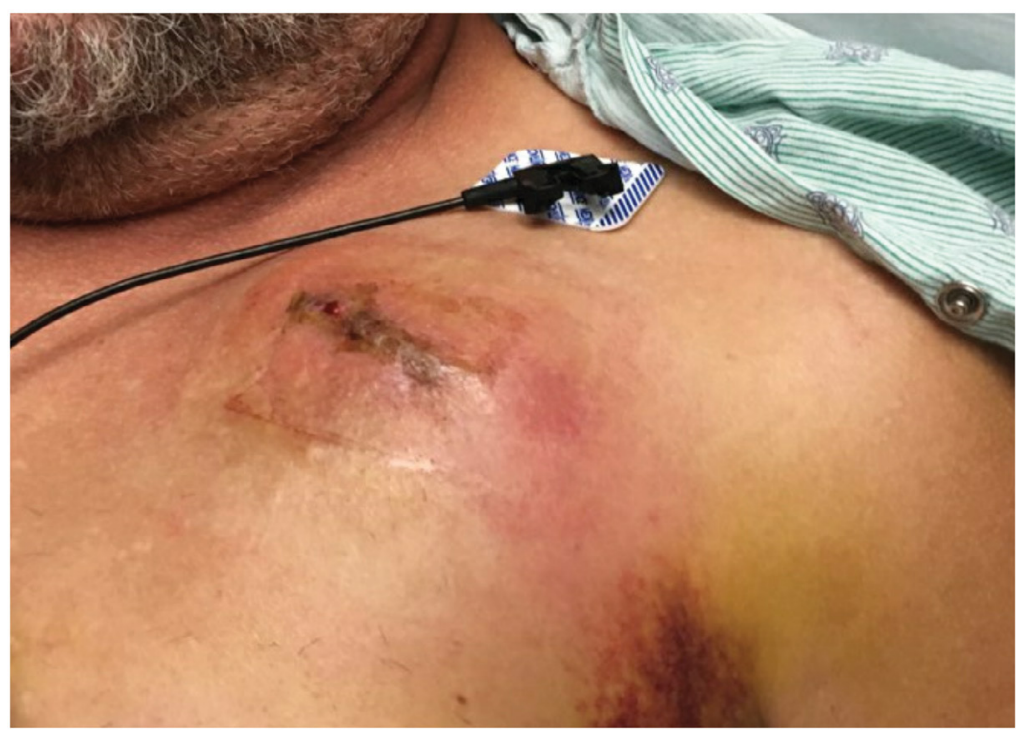

Figure 1: Clinical Presentation: Patient presented with complaint of fever, pain, and swelling at device site. On exam, there was incision site drainage, erythema, induration and ecchymosis extending into the axilla, accompanied by warmth on palpation.

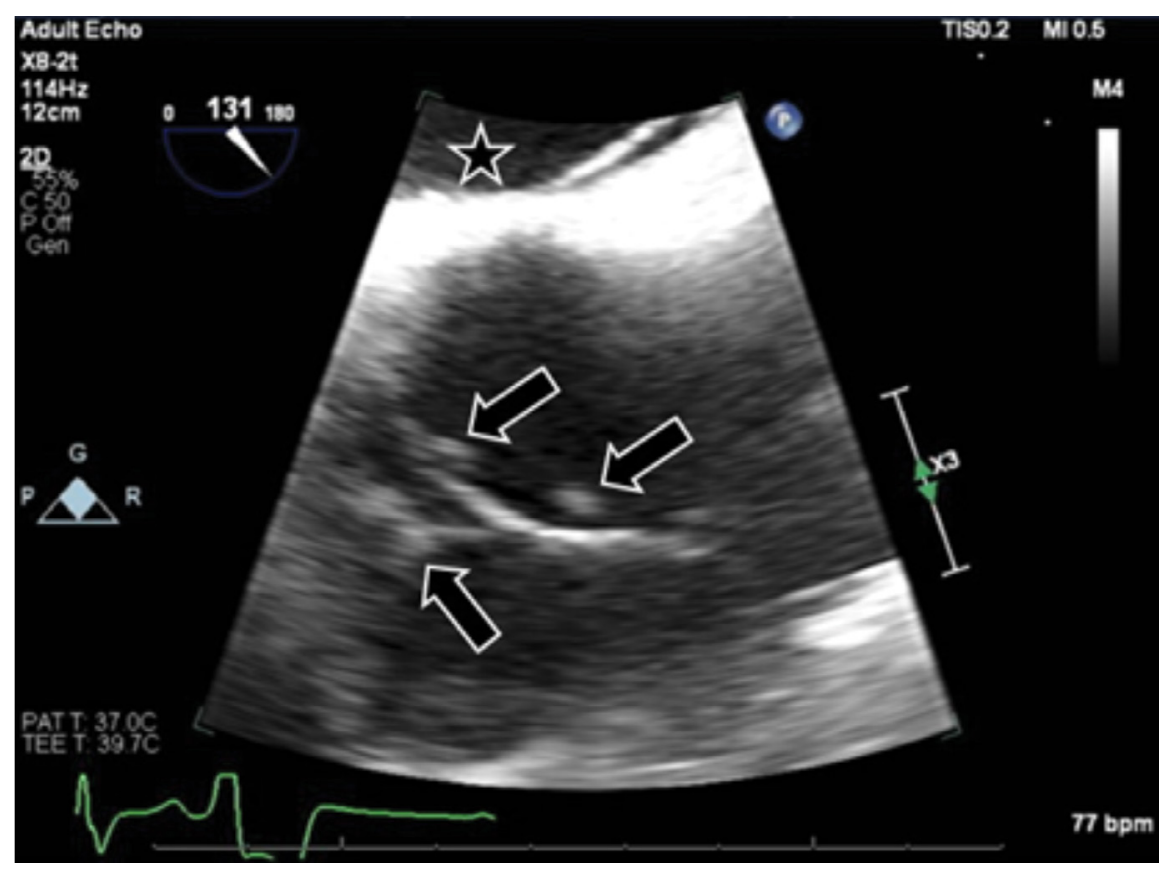

Figure 2: Transesophageal Echocardiogram: Transesophageal echocardiogram in modified bicaval view showing multiple vegetations (arrows) on right atrial lead near junction of inferior vena cava. In this focus image, the left atrium is labeled (star).

gnancy, connective tissue diseases, or drug reaction.

Lab work revealed elevated lactic acid to $2.31 \mathrm{mmol} / \mathrm{L}$ (normal range, $0.5-2.2 \mathrm{mmol} / \mathrm{L}$ ), peripheral white blood cell count was $8,190 /$ uL with $74.7 \%$ neutrophils. Blood cultures were collected, with the initial set was negative for any growth. Device pocket was explored on the second day of admission and showed evidence of pocket hematoma, but no significant purulence or necrotic tissue was noted. The decision to keep the device in place was made, and the pocket was irrigated and sutured closed. Wound cultures were negative for routine aerobic and anaerobic organisms. The patient was initially started on intravenous vancomycin, but continued to have intermittent fevers following pocket exploration. Repeat blood cultures were collected, and a TEE was obtained. Repeat blood cultures were positive for acidfast bacillus presumed to be a mycobacterium, though initially the species identification was not known. TEE showed vegetations on the right atrial lead of the CRT-D and at the junction of the right atrium and inferior vena cava (Figure 2).

Following discovery of the mycobacteremia and cardiac device vegetations, the CRT-D was extracted, and the patient was started on amikacin, imipenem-cilasta- 
tin, and linezolid, with cessation of his fevers and improvement of the purulent drainage, erythema, and tenderness at the device pocket site. The leads were sent for culture and revealed $2+$ acid-fast bacilli on smear, later speciated as Mycobacterium abscessus by MALDI-TOF (Matrix Assisted Laser Desorption/Ionization Time of Flight) Mass Spectrometry. Infectious disease was consulted, the patient was discharged on home IV amikacin, imipenem, and oral linezolid therapy with close follow up. The patient was also noted to have deep vein thrombosis of left axillary and brachial veins, as well as superficial venous thrombosis of his left cephalic and basilic veins, and was discharged home on apixaban.

Patient had clinical improvement following removal of the CRT-D and being initiated on initial IV antibiotic regimen. After discharge, culture susceptibilities of the Mycobacterium abscessus showed resistance to several antibiotics including Linezolid, Clarithromycin, and Imipenem. At outpatient follow up with Infectious Disease, patient reported ongoing generalized weakness, lethargy, and intermittent drainage of his pacemaker pocket. He was subsequently admitted to the hospital for re-initiation of IV antibiotics, specifically Eravacycline and Amikacin. He completed 8 weeks of the aforementioned regimen with subsequent improvement of his symptoms. Patient will be re-evaluated for placement of additional CRT-D at a future date.

\section{Discussion}

Our patient had his cardiac device in place for only 12 days at presentation with local and systemic infection symptoms. Non-tuberculous, rapid-growing Mycobacterial infections of implanted cardiac devices generally occur in patients with long-indwelling intravascular devices, presumably through hematogenous seeding of the device as opposed to contiguous spread. They tend to occur more than 6 months after device manipulation [2]. Most early cardiac device infections are characteristically pyogenic, with $>90 \%$ being caused by Staphylococcus aureus, coagulase-negative Staphylococci, or other skin gram positive bacteria including Streptococci [5]. For the afore mentioned reasons, in addition to the fact that rapid-grower colonies take up to 14 days to grow, the diagnosis of cardiac device infections with rapid-growers require a heightened index of suspicion.

Given the paucity of cases, lack of evidence from randomized controlled studies, and the considerable innate resistance of $M$. abscessus, first-line antimicrobial regimens are not well established. In similar cases of severe and disseminated $M$. abscessus infection, patients require intensive induction therapy with a combination of at least three empiric antimicrobials, including clarithromycin, amikacin, cefoxitin, imipenem-cilastatin, linezolid, or tigecycline. This is complicated by increasing macrolide resistance, high risk of unintended toxicity, narrow therapeutic windows, and lack of oral options with high bioavailability [3]. Once the susceptibilities of the individual pathogen are known, the antimicrobial regimen can be tailored in the continuation phase, and often continued for 24 weeks or longer [3,4,6-9].

We believe the patient's infection was acquired from the local environment, via contamination of the wound followed by contiguous spread from the pocket to the vascular compartment. Previous studies in the state of Texas suggest that rapidly-growing Mycobacterium were found in the local water supply and hospital dust $[6,7]$. It is possible the patient acquired the infection from showering after implantation of the device, secondary to the surgical dressing not being an adequate enough barrier to prevent water penetration into the surgical site. Future studies could focus on appropriate wound care and surgical dressing in prevention of nontuberculous Mycobacterial infections.

We present a case of a patient presenting with Mycobacterium abscessus infectious endocarditis secondary to lead vegetation on a recently implanted CRT-D, ultimately requiring removal of the device and an extended course of antibiotic therapy. In a patient presenting with concern for infectious endocarditis, or implantable cardiac device infection, a thorough investigation to evaluate for infection is paramount, and it is important to consider atypical organisms when more typical organisms have been excluded. Furthermore, it may be worth considering treating patients at higher risk of developing atypical infections with an appropriate prophylactic antibiotic therapy after device implant.

\section{Conflicts of Interest}

All authors have no conflicts of interest to disclose.

\section{Funding}

None.

\section{References}

1. Al-Ghamdi B, Widaa HE, Shahid MA, Aladmawi M, Alotaibi $\mathrm{J}$, et al. (2016) Cardiac implantable electronic device infection due to Mycobacterium species: A case report and review of the literature. BMC Res Notes 9: 414.

2. Phadke VK, Hirsh DS, Goswami ND (2016) Patient report and review of rapidly growing mycobacterial infection after cardiac device implantation. Emerg Infect Dis 22: 389-395.

3. Kessler AT, Kourtis AP (2004) Mycobacterium abscessus as a cause of pacemaker infection. Med Sci Monit 10: CS60-CS62.

4. Zhu J, Yang Q, Pan J, Shi H, Jin B, et al. (2019) Cardiac resynchronization therapy-defibrillator pocket infection caused by Mycobacterium fortuitum: A case report and review of the literature. BMC Cardiovasc Disord 19: 53.

5. Sandoe JAT, Barlow G, Chambers JB, Gammage M, Guleri A, et al. (2015) Guidelines for the diagnosis, prevention and management of implantable cardiac electronic device infection. Report of a joint Working Party project on behalf of the British Society for Antimicrobial Chemotherapy (BSAC, host organization), British Heart Rhythm Society (BHRS), 
British Cardiovascular Society (BCS), British Heart Valve Society (BHVS) and British Society for Echocardiography (BSE). J Antimicrob Chemother 70: 325-359.

6. Wallace RJ Jr, Musser JM, Hull SI, Silcox VA, Steele LC, et al. (1989) Diversity and sources of rapidly growing mycobacteria associated with infections following cardiac surgery. J Infect Dis 159: 708-716.

7. Cutay AM, Horowitz HW, Pooley RW, Van Horn K, Wormser GP (1998) Infection of epicardial pacemaker wires due to Mycobacterium abscessus. Clin Infect Dis 26: 520-521.
8. Baddour LM, Epstein AE, Erickson CC, Knight BP, Levison ME, et al. (2010) Update on cardiovascular implantable electronic device infections and their management: A scientific statement from the American Heart Association. Circulation 121: 458-477.

9. Hu YL, Bridge B, Wang J, Jovin IS (2012) Mycobacterium fortuitum causing infection of a biventricular pacemaker/ implantable cardioverter defibrillator. Int J Mycobacteriol 1: 221-223. 